\title{
Study of University Students' Stress Accommodation and Participation of Leisure Sports
}

\author{
Yi Fang \\ Institute of Physical Education, Guangxi Normal University, Guilin, Guangxi, China
}

\begin{abstract}
By the method of questionnaire consultation, this article probes into the relation between university students' stress accommodation and participation of leisure sports. The result indicates that different university students' attribute and the pressure source contain obvious and sexual difference. The University students' pressure source indexes involve the recreation exercise participates the factor and the pressure index contain obvious difference, representing university students' participation of leisure sports and stress are closely related.
\end{abstract}

Keywords - Leisure sports, Stress accommodation, Participation behavior

\section{大学生压力调控与休闲运动参与的关联性研究}

易芳

广西师范大学 体育学院, 桂林, 广西, 中国

摘 要 通过问卷调查法, 探究大学生压力调控与休闲运动参与的关联性。结果表明: 不同大学生属性与压力来源有显著性的差 异。大学生压力来源指标可归纳为五个方面: 环境生活适应力、物质与两性交往判断力、自我生活适应力、自我价值判断力和学习适 应力。大学生休闲运动参与因素与压力指标有显著性差异, 表明大学生休闲运动参与与压力有密切的关联性。

关键词 休闲运动, 压力调控, 参与行为

1. 引言

在我国快速发展的过程中, 具有热情、潜力的青少年 是社会发展及持续进步的原动力, 青少年常扮演着社会引 领者角色，处于青少年后期的大学生完成学业后即步入社 会成为国家的栋梁, 但近年来大学生自杀、忧郁等负面事 件却不断攀升, 青少年身心健康问题层出不穷。心理压力 (以下简称 “压力”) 问题是现代社会十分关注的问题, 一定程度的压力有助于提高个人学习、工作的效率, 但过 度的压力则会影响身心健康。众所周知，体育运动是促进 人们身心健康的重要手段之一。因此, 是否可以将大学生 身心健康问题归因于他们不知如何安排休闲时间, 发泄过 剩精力? 或者是大学生价值观的改变受不正当观念的影

响? 这些都有其研究的必要。本研究旨在了解大学生参与 休闲运动的情况与差异, 及与压力调控的相关程度, 研究 目的为: 1. 探讨大学生压力来源与休闲运动参与程度。 2 . 建构大学生参与休闲运动与压力指标来源的关联性。3. 分 析大学生休闲运动参与程度与压力程度的关联性。本研究
对于了解当今大学生压力现状, 进一步指导大学生积极有 规律地参与休闲运动, 有效缓解大学生的压力, 促进其身 心健康发展, 防止不幸事件的发生, 有着重要的意义。

\section{2. 研究方法}

2.1 问卷设计

本研究内容包括: 1 . 研究对象基本资料: 性别、年龄、 父母管教方式、年级、户籍地、家庭月收入 6 个变量。 2 . 参与运动休闲类型及频率变量。3. 根据杨莉莉、张霞、江 雷等 [1-6]研究文献归纳压力来源为家庭、学校、人际、自 我方面及两性交往等五方面, 制定 40 个压力来源因素作为 研究变量, 并使用李克特态度量表, 将大学生考虑条件的 评级分成完全不同意至完全同意 6 个等级。

\section{2 样本选取与抽样方法}

抽样对象为广西师范大学三、四年级学生, 抽样方式 采用分层随机抽样方式 (共 22 个院系)。三年级发放问卷 
为 700 份, 回收 502 份, 经整理扣除无效问卷, 有效问卷 为 483 份。四年级发放问卷为 400 份, 回收 271 份, 经整 理扣除无效问卷, 有效问卷为 238 份。有效问卷总计 721 份。采用结构型问卷, 让受访者对本研究设计的主题以指 定问题的相关选项的回答方式来回答。

\section{3 分析方法}

调查问卷回收后, 将问卷的填答数据输入电脑, 使用 SPSS13. 0 统计软件, 对数据进行描述性统计分析、满意度 实际与预期差距分析和成对样本 $\mathrm{T}$ 检验。

\section{3. 研究结果与讨论}

\section{1 研究对象基本资料（表 1)}

表 1 研究对象基本资料表

\begin{tabular}{|c|c|c|c|}
\hline \multicolumn{2}{|c|}{ 基本资料 } & 人数 & 百分比 (\%) \\
\hline \multirow{2}{*}{ 性别 } & 男性 & 192 & 26.6 \\
\hline & 女性 & 529 & 73.4 \\
\hline \multirow{3}{*}{ 年龄 } & 18-20 岁 & 126 & 17.5 \\
\hline & 21-25 岁 & 581 & 80.6 \\
\hline & 25 岁以上 & 14 & 1.9 \\
\hline \multirow{4}{*}{$\begin{array}{l}\text { 管教 } \\
\text { 方式 }\end{array}$} & 开明民主 & 618 & 85.7 \\
\hline & 专制权威 & 65 & 9.0 \\
\hline & 纵容溺爱 & 14 & 1.9 \\
\hline & 其他 & 24 & 3.3 \\
\hline \multirow{2}{*}{ 年级 } & 三年级 & 483 & 67.0 \\
\hline & 四年级 & 238 & 33.0 \\
\hline \multirow{6}{*}{$\begin{array}{c}\text { 家庭 } \\
\text { 月收入 }\end{array}$} & 5000 元以下 & 180 & 25.0 \\
\hline & 5000-10000 & 312 & 43.3 \\
\hline & $10000-20000$ & 122 & 16.9 \\
\hline & $20000-30000$ & 61 & 8.5 \\
\hline & $30000-40000$ & 15 & 2.1 \\
\hline & 40000 元以上 & 31 & 4.3 \\
\hline
\end{tabular}

\section{2 大学生对压力来源认知的描述性统计分析}

经过分析后, 本研究发现影响大学生对压力来源认知 的因素中, 整体而言, 受访者都支持本研究所拟的因素, 是大学生面对压力来源所应注意的因素 (表 2)。
表 2 大学生对压力来源认知的描述性统计分析表

\begin{tabular}{|c|c|c|c|}
\hline 压力来源 & 平均数 & 标准差 & 排名 \\
\hline 父母亲死亡 & 3.38 & 1.20 & 1 \\
\hline 家庭经济有困难 & 3.28 & 0.91 & 2 \\
\hline 父母亲离婚或分居 & 3.21 & 1.12 & 3 \\
\hline 担心学习考试 & 3.20 & 1.04 & 4 \\
\hline 有些课程听不懂老师上课的内容 & 3.18 & 1.10 & 5 \\
\hline 对某科课程不感兴趣 & 3.16 & 1.13 & 6 \\
\hline 父母亲对我的期望过高 & 3.09 & 0.90 & 7 \\
\hline 怀疑自己的能力不足 & 3.03 & 1.13 & 8 \\
\hline 对自己期望过高 & 3.01 & 1.02 & 9 \\
\hline 因考试不及格需要重修 & 2.99 & 1.22 & 10 \\
\hline 没有足够的零用钱或物质享受 & 2.96 & 1.16 & 11 \\
\hline 朋友生重病或去世 & 2.87 & 1.31 & 12 \\
\hline 觉得自己外表 (相貌、身材) 比不上别人 & 2.78 & 1.11 & 13 \\
\hline 与异性朋友吵架或分手 & 2.73 & 1.25 & 14 \\
\hline 因交往异性朋友而造成自己情绪上的不稳 & 2.69 & 1.17 & 15 \\
\hline 喜欢某位异性朋友但不敢表达 & 2.68 & 1.18 & 16 \\
\hline 初次与自己喜欢的异性朋友交往 & 2.68 & 1.21 & 17 \\
\hline 父母亲管我太严格太多 & 2.67 & 0.98 & 18 \\
\hline 家中有人严重生病或身体不适 & 2.67 & 1.25 & 19 \\
\hline 与父母或兄弟姐妹发生争执或冲突 & 2.66 & 1.14 & 20 \\
\hline 父母亲对我的管教方式不一致 & 2.66 & 1.03 & 21 \\
\hline 因疾病或意外使健康状况有所改变 & 2.66 & 1.16 & 22 \\
\hline 校园暴力事件曾发生在我身上 & 2.64 & 1.24 & 23 \\
\hline 与要好的朋友关系结束 & 2.64 & 1.36 & 24 \\
\hline 喜欢某位异性朋友但被拒绝 & 2.63 & 1.23 & 25 \\
\hline 父母反对我交异性朋友 & 2.59 & 1.19 & 26 \\
\hline 学校管教太严格 & 2.58 & 0.99 & 27 \\
\hline 与异性朋友相处会不自在 & 2.57 & 1.13 & 28 \\
\hline 因交往异性朋友而与其他朋友、同学疏远 & 2.57 & 1.12 & 29 \\
\hline 因某些严重的问题或过失被记过 & 2.56 & 1.17 & 30 \\
\hline 异性朋友太多不知如何选择 & 2.55 & 1.16 & 31 \\
\hline 家庭居住环境不佳 & 2.54 & 1.01 & 32 \\
\hline 违反校规, 被学校处罚 & 2.50 & 1.08 & 33 \\
\hline 父母亲发生争吵 & 2.49 & 1.13 & 34 \\
\hline 因交往异性朋友而使自己的成绩退步 & 2.48 & 1.06 & 35 \\
\hline 班上同学学习竞争风气太激烈 & 2.47 & 1.06 & 36 \\
\hline 父母反对我所交往的朋友 & 2.39 & 1.21 & 37 \\
\hline 被同学或朋友指责、批评 & 2.35 & 1.25 & 38 \\
\hline 与同辈或朋友的人际关系欠佳 & 2.32 & 1.22 & 39 \\
\hline 与同学或朋友发生争吵 & 2.32 & 1.20 & 40 \\
\hline
\end{tabular}


受访者认为在面对压力来源问题时, 最同意的因素前 五项依据平均数为父母亲死亡 (3.3750)、家庭经济有困难 (3.2837)、父母亲离婚或分居（3.2057）、担心学校考试 (3.1963)、有些课程听不懂老师上课的内容（3.1833）。 平均数较低的, 也是受访者认为在面对压力来源问题时同 意得较少的因素, 前五项依次是班上同学学习竞争风气太 激烈（2.4658）、父母反对我所交往的朋友（2.3918）、被 同学或朋友指批评 (2.3462)、与同辈或朋友的人际关系欠 佳 (2.3233)、与同学或朋友发生争吵 (2.3194)。

\section{3 影响大学生参与休闲运动因素的描述性统计分析}

研究结果表明, 影响大学生参与休闲运动的因素中, 受访者 都支持本研究拟出的因素为所应注意的项目 (表 3 )。受访者认为 在参与休闲运动时, 会影响参与的因素里, 最同意的前五项依据 平均数为可放松情绪与纾解压力 (3.6143)、时间可以配合需求 (3.5974)、促进身体的健康（3.5178）、可及性高且交通便利 (3.5078)、保持身材 (3.4595)。

表 3 影响大学生参与休闲运动因素的描述性统计分析表

\begin{tabular}{|c|c|c|c|}
\hline 影响因素 & 平均数 & 标准差 & 排名 \\
\hline 可放松情绪与纾解压力 & 3.61 & 1.82 & 1 \\
\hline 时间可以配合需求 & 3.60 & 1.83 & 2 \\
\hline 促进身体的健康 & 3.52 & 1.81 & 3 \\
\hline 可及性高且交通便利 & 3.51 & 1.83 & 4 \\
\hline 保持身材 & 3.46 & 1.81 & 5 \\
\hline 拓展社交能力 & 3.25 & 1.74 & 6 \\
\hline 提升生活品质 & 3.24 & 1.73 & 7 \\
\hline $\begin{array}{c}\text { 家人或朋友也事此项休 } \\
\text { 闲运动 }\end{array}$ & 3.22 & 1.76 & 8 \\
\hline 离家近 & 3.20 & 1.77 & 9 \\
\hline 增进家庭情感交流 & 3.17 & 1.74 & 10 \\
\hline 学习运动技巧 & 3.07 & 1.70 & 11 \\
\hline
\end{tabular}

3.4 大学生对压力调控认知的因素分析

在大学生对压力调控的认知因素方面, 通过归类分析, 区分为五个主要且显著解释变异的因子 (表 4)。

指标一: “自我生活适应力”, 包括 “与父母或兄弟姐 妹发生争执或冲突”、“父母亲对我的期望过高”、“父母亲 管我太严格及太多”、“父母亲发生争吵”、“父母亲对我的 管教方式不一致”、“家庭居住环境不佳”、“学校管教太严 格”、“被同学或朋友指责、批评”、“与同学或朋友发生争 吵”、“与同辈或朋友的人际关系欠佳”、“对自己期望过高”、 “因疾病或意外使健康状况有所改变”、“因某些严重的问 题或过失被记过” 等。

指标二: “物质与两性交往判断力”, 包括 “没有足够 的零用钱或物质享受”、“喜欢某位异性朋友但不敢表达”、
“因交往异性朋友而造成自己情绪上的不稳”、“父母反对 我交异性朋友”、“”喜欢某位异性朋友但被拒绝、“初次与 自己喜欢的异性朋友交往”、“因交往异性朋友而与其他朋 友、同学疏远”、“异性朋友太多不知如何选择”、“因交往 异性朋友而使自己的成绩退步” 等。

指标三: “环境生活适应力”, 包括 “家庭经济困难”、 “家中有人严重生病或身体不适”、“父母章离婚或分居”、 “父母亲死亡”、“父母反对我所交往的朋友”、“与要好的 朋友关系结束”、“朋友生重病或去世”、“与异性朋友吵架 或分手”等。

指标四: “自我价值判断力”, 包括 “对某科课程不感 兴趣”、“有些课程听不懂老师上课的内容”、“担心学校考 试”、“觉得自己外表（相貌、身材）比不上别人”、“怀疑 自己的能力不足”、“与异性朋友相处会不自在” 等。

指标五: “学习适应力”, 包括 “因成绩不及格需要重 修”、“班上同学学习竞争风气太激烈”、“违反校规, 被学 校处罚”、“校园暴力事件曾发生在我身上” 等。

\section{4. 大学生压力调控与休闲运动参与的认知与看法和 个人属性显著性分析}

\section{1 大学生个人属性不同与压力来源的显著性差异分析}

表 5 分析结果显示, 在性别方面, 对 “父母亲对我的 期望过高”、“父母管我太严格太多”、“父母亲对我的管教 方式不一致”、“异性朋友太多不知如何选择” 的压力来源 有高度显著性差异, 对 “与父母或兄弟姐妹发生争执或冲 突” 的压力来源有非常显著性差异, 其余的压力来源仅有 显著性差异; 在年龄方面, 对 “与异性朋友相处会不自在”、 “因交往异性朋友而使自己的成绩退步” 的压力来源有非 常显著性差异, 其余的压力来源仅有显著性差异; 在年级 方面, 对 “有些课程听不懂老师上课的内容”、“担心学校 考试”、“没有足够的零用钱或物质享受” 的压力来源有非 常显著性差异, 其余的压力来源仅有显著性差异; 在管教 方面, 对 “父母亲管我太严格太多” 的压力来源有高度显 著性差异, 对 “父母亲对我的期望过高”、“对某科课程不 感兴趣”、“校园暴力事件曾发生在我身上” 的压力来源有 非常显著性差异, 其余的压力来源仅有显著性差异; 在家 庭月收入方面, 对 “家庭经济有困难”、“与父母或兄弟姐 妹发生争执或冲突”、“没有足够的零用钱或物质享受” 的 压力来源有高度显著性差异, 对 “与要好的朋友关系结束”、 “初次与自己喜欢的异性朋友交往”、“因交往异性朋友而 与其他朋友、同学疏远”、“异性朋友太多不知如何选择” 的压力来源有非常显著性差异, 其余的压力来源仅有显著 性差异。 
表 4 大学生压力指标建构表

\begin{tabular}{|c|c|c|c|c|c|}
\hline 压力指标, 压力来源 & 自我生活适应力 & 物质欲两性交往判断力 & 环境生活适应力 & 自我价值判断力 & 学习适应力 \\
\hline 与父母或兄弟发生争执或冲突 & 0.86 & 0.37 & 0.46 & 0.49 & 0.44 \\
\hline 父母亲对我的期望过高 & 0.70 & 0.61 & 0.68 & 0.42 & 0.36 \\
\hline 父母亲管我太严格及太多 & 0.77 & 0.55 & 0.55 & 0.73 & 0.56 \\
\hline 父母亲发生争吵 & 0.86 & 0.45 & 0.53 & 0.54 & 0.61 \\
\hline 父母亲对我的管教方式不一致 & 0.80 & 0.53 & 0.41 & 0.73 & 0.49 \\
\hline 家庭居住环境不佳 & 0.87 & 0.40 & 0.50 & 0.61 & 0.51 \\
\hline 学校管教太严格 & 0.68 & 0.61 & 0.25 & 0.61 & 0.66 \\
\hline 被同学或朋友指责、批评 & 0.78 & 0.40 & 0.63 & 0.41 & 0.63 \\
\hline 与同学或朋友发生争吵 & 0.83 & 0.49 & 0.70 & 0.42 & 0.71 \\
\hline 与同辈或朋友的人际关系欠佳 & 0.82 & 0.49 & 0.71 & 0.50 & 0.70 \\
\hline 对自己期望过高 & 0.83 & 0.60 & 0.50 & 0.38 & 0.47 \\
\hline 因疾病或意外使健康状况有所改变 & 0.77 & 0.56 & 0.63 & 0.33 & 0.72 \\
\hline 因某些严重的问题或过失被记过 & 0.71 & 0.69 & 0.53 & 0.42 & 0.89 \\
\hline 家庭经济有困难 & 0.56 & 0.56 & 0.76 & 0.37 & 0.32 \\
\hline 家中有人严重生病或身体不适 & 0.64 & 0.41 & 0.88 & 0.51 & 0.54 \\
\hline 父母亲离婚或分居 & 0.69 & 0.51 & 0.85 & 0.35 & 0.58 \\
\hline 父母亲死亡 & 0.46 & 0.53 & 0.84 & 0.39 & 0.60 \\
\hline 父母反对我所交往的朋友 & 0.58 & 0.53 & 0.81 & 0.59 & 0.53 \\
\hline 与要好的朋友关系结束 & 0.48 & 0.50 & 0.83 & 0.61 & 0.42 \\
\hline 朋友生重病或去世 & 0.50 & 0.60 & 0.90 & 0.49 & 0.53 \\
\hline 与异性朋友吵架或分手 & 0.37 & 0.69 & 0.71 & 0.37 & 0.48 \\
\hline 对某科课程不感兴趣 & 0.54 & 0.49 & 0.33 & 0.88 & 0.54 \\
\hline 有些课程听不懂老师上课的内容 & 0.56 & 0.58 & 0.61 & 0.84 & 0.46 \\
\hline 担心学校考试 & 0.54 & 0.54 & 0.64 & 0.82 & 0.64 \\
\hline 觉得外表 (相貌、身材) 比不上别人 & 0.69 & 0.55 & 0.58 & 0.69 & 0.56 \\
\hline 怀疑自己的能力不足 & 0.55 & 0.59 & 0.62 & 0.79 & 0.47 \\
\hline 与异性朋友相处不自在 & 0.48 & 0.74 & 0.71 & 0.75 & 0.43 \\
\hline 因考试不及格需要重修 & 0.67 & 0.61 & 0.66 & 0.47 & 0.82 \\
\hline 班上同学学习的竞争风气太激烈 & 0.63 & 0.58 & 0.49 & 0.71 & 0.78 \\
\hline 违反校规，被学校处罚 & 0.48 & 0.49 & 0.58 & 0.62 & 0.84 \\
\hline 校园暴力事件曾发生在我身上 & 0.53 & 0.40 & 0.65 & 0.64 & 0.85 \\
\hline 没有足够的零用钱或物质享受 & 0.63 & 0.69 & 0.44 & 0.35 & 0.30 \\
\hline 喜欢某位异性朋友但不敢表达 & 0.48 & 0.93 & 0.57 & 0.52 & 0.49 \\
\hline 因交往异性朋友而造成自己情绪上的不稳 & 0.43 & 0.79 & 0.60 & 0.67 & 0.42 \\
\hline 父母反对我交异性朋友 & 0.53 & 0.89 & 0.60 & 0.64 & 0.44 \\
\hline 喜欢某位异性朋友但被拒绝 & 0.40 & 0.88 & 0.61 & 0.65 & 0.41 \\
\hline 初次与自己喜欢的异性朋友交往 & 0.42 & 0.76 & 0.64 & 0.59 & 0.65 \\
\hline 因交往异性朋友而与其他朋友、同学疏远 & 0.60 & 0.77 & 0.52 & 0.47 & 0.73 \\
\hline 异性朋友太多不知如何选择 & 0.51 & 0.88 & 0.45 & 0.37 & 0.58 \\
\hline 因交往异性朋友而使自己成绩退步 & 0.50 & 0.83 & 0.39 & 0.52 & 0.68 \\
\hline
\end{tabular}

注: 提取方法为主成分分析; 利用最大变异法 (varimax) 进行转轴分析, 其特征值累积解释度 $79.002 \%$ 。 
表 5 大学生个人属性与压力来源的显著性差异分析表

\begin{tabular}{|c|c|c|}
\hline 个人属性 & 压力来源 & 统计数值 \\
\hline \multirow{10}{*}{ 性＼cjkstart别 } & 与父母或兄弟发生争执或冲突 & $0.04 * *$ \\
\hline & 父母亲对我的期望过高 & $0.00 * * *$ \\
\hline & 父母亲管我太严格太多 & $0.00 * * *$ \\
\hline & 父母亲对我的管教方式不一致 & $0.00 * * *$ \\
\hline & 对某科课程不感兴趣 & $0.02 *$ \\
\hline & 因考试不及格需要重修 & $0.02 *$ \\
\hline & 因疾病或意外使健康状况有所改变 & $0.03 *$ \\
\hline & 因某些严重的问题或过失被记过 & $0.01 *$ \\
\hline & 喜欢某位异性朋友但被拒绝 & $0.01 *$ \\
\hline & 异性朋友太多不知如何选择 & $0.00 * * *$ \\
\hline \multirow{4}{*}{ 年 龄 } & 没有足够的零用钱或物质享受 & $0.03 *$ \\
\hline & 因交往异性朋友而造成自己情绪上的不稳 & $0.03 *$ \\
\hline & 与异性朋友相处会不自在 & $0.00 * *$ \\
\hline & 因交往异性朋友而使自己的成绩退步 & $0.01 * *$ \\
\hline \multirow{4}{*}{ 级 } & 有些课程听不懂老师上课的内容 & $0.00 * *$ \\
\hline & 担心学校考试 & $0.01 * *$ \\
\hline & 没有足够的零用钱或物质享受 & $0.00 * *$ \\
\hline & 因交往异性朋友而造成自己情绪上的不稳 & $0.03 *$ \\
\hline \multirow{12}{*}{ 管教方式 } & 父母亲对我的期望过高 & $0.01 * *$ \\
\hline & 父母亲管我太严格太多 & $0.00 * * *$ \\
\hline & 父母亲对我的管教方式不一致 & $0.01 \%$ \\
\hline & 对某科课程不感兴趣 & $0.01 * *$ \\
\hline & 校园暴力事件曾发生在我身上 & $0.01 * *$ \\
\hline & 父母反对我所交往的朋友 & $0.03 *$ \\
\hline & 怀疑自己的能力不足 & $0.04 *$ \\
\hline & 没有足够的零用钱或物质享受 & $0.03 *$ \\
\hline & 因交往异性朋友而造成自己情绪上的不稳 & $0.03 *$ \\
\hline & 与异性朋友相处会不自在 & $0.03 *$ \\
\hline & 父母反对我交异性朋友 & $0.05 *$ \\
\hline & 异性朋友太多不知如何选择 & $0.04 *$ \\
\hline \multirow{21}{*}{ 家庭月收入 } & 家庭经济有困难 & $0.00 * * *$ \\
\hline & 与父母或兄弟发生争执或冲突 & $0.02 * * *$ \\
\hline & 父母亲发生争吵 & $0.01 *$ \\
\hline & 父母亲对我的管教方式不一致 & $0.00 *$ \\
\hline & 家庭居住环境不佳 & $0.00 *$ \\
\hline & 家中有人严重生病或身体不适 & $0.03 *$ \\
\hline & 父母亲死亡 & $0.03 *$ \\
\hline & 与要好的朋友关系结束 & $0.10 * *$ \\
\hline & 朋友生重病或去世 & $0.05 *$ \\
\hline & 被同学或朋友指责、批评 & $0.02 *$ \\
\hline & 与同学或朋友发生争吵 & $0.03 *$ \\
\hline & 觉得自己外表 (相貌、身材) 比不上别人 & $0.03 *$ \\
\hline & 怀疑自己的能力不足 & $0.02 *$ \\
\hline & 没有足够的零用钱或物质享受 & $0.00 * * *$ \\
\hline & 对自己期望过高 & $0.04 *$ \\
\hline & 父母反对我交异性朋友 & $0.04 *$ \\
\hline & 与异性朋友吵架或分手 & $0.02 *$ \\
\hline & 初次与自己喜欢的异性朋友交往 & $0.01 * *$ \\
\hline & 因交往异性朋友而与其他朋友、同学疏远 & $0.00 * *$ \\
\hline & 异性朋友太多不知如何选择 & $0.01 * *$ \\
\hline & 因交往异性朋友而使自己的成绩退步 & $0.02 *$ \\
\hline
\end{tabular}

注: *p<.05, ** $\mathrm{p}<.01, * * * \mathrm{p}<.001$.

\section{2 大学生个人属性不同与压力程度的显著性差异分析}

本研究调查经分析后, 得出下列几项结果 (表 6): 受 访者性别的不同除对 “物质与两性交往判断力”、“学习适 应力” 有高度显著性的差异, 对 “环境生活适应力” 有显 著性差异外，其余则无显著性的差异; 受访者年龄的不同 对 “自我生活适应力”、“自我价值判断力” 有显著性差异, 其余都无显著性的差异; 受访者父母管教方式的不同对“物 质与两性交往判断力” 有高度显著性差异, 对 “环境生活 适应力” 有非常显著性差异, 其余都无显著性差异; 受访 者家庭月收入的不同对 “环境生活适应力” 有高度显著性 差异, 对 “学习适应力” 有显著性差异, 其余都无显著性 差异。

表 6 大学生个人属性不同于压力程度的显著性差异分析表

\begin{tabular}{|c|c|c|c|c|c|}
\hline $\begin{array}{c}\text { 分类项目 } \\
\text { 个人属性 }\end{array}$ & $\begin{array}{c}\text { 自我生活 } \\
\text { 适应力 }\end{array}$ & $\begin{array}{c}\text { 物质与两性 } \\
\text { 交往判断力 }\end{array}$ & $\begin{array}{c}\text { 环境生活 } \\
\text { 适应力 }\end{array}$ & $\begin{array}{c}\text { 自我价值 } \\
\text { 判断力 }\end{array}$ & $\begin{array}{c}\text { 学习适 } \\
\text { 应力 }\end{array}$ \\
\hline 性 别 & 0.55 & $0.00 * * *$ & $0.03 *$ & 0.11 & $0.00 * * *$ \\
\hline 年 龄 & $0.02 *$ & 0.17 & 0.69 & $0.05 *$ & 0.21 \\
\hline 管教方式 & 0.17 & $0.00 * * *$ & $0.01 * *$ & 0.11 & 0.38 \\
\hline 年 级 & 0.14 & 0.47 & 0.18 & 0.32 & 0.07 \\
\hline 家庭月收入 & 0.44 & 0.7 & $0.00 * * *$ & 0.11 & $0.02 *$ \\
\hline
\end{tabular}

注: $* \mathrm{p}<.05, * * \mathrm{p}<.01, * * * \mathrm{p}<.001$ 。

\section{5. 结论与建议}

\section{1 结论}

1)大学生个人属性不同与压力来源有不同的显著性差 异, 代表不同的压力来源会随着大学生个人的性别、年龄、 年级、父母管教方式、家庭月收入有不同的调控方式, 说 明目前大学生在各种环境中必须面对不同的压力来源, 而 且要尽力适应。

2）在休闲运动参与的 11 项因素中, 大学生认为 “可 及性高且交通便利”、“时间可以配合需求”、“学习运动技 巧”、“增进家庭情感交流”、“家人或朋友也从事此项休闲 运动”、“提升生活品质”、“拓展社交能力”、“可放松情绪 与纾解压力” 是调控各种压力, 参与休闲运动最具显著性 的 4 项因素。

3）大学生个人属性不同在面对压力程度时, 对 “自我 生活适应力”、“物质与两性交往判断力”、“环境生活适应 力”、“自我判断力”、“学习适应力” 都有不同程度的显著 性差异, 符合本研究所归类的家庭、学校、人际关系、自 我方面、两性交往方面的压力来源要素。 


\section{2 建议}

1) 本研究发现大学生面对压力来源时, 会影响大学生 从事休闲运动考虑运动设施的交通便利性。因此, 学校、 班级应以统一组织或体育俱乐部组织等形式, 促进大学生 休息运动的参与, 或者与邻近学习合作, 长时间开放休闲 运动设施, 提供使用的便利性, 满足大学生参与休闲运动 的需求, 以减少压力来源。

2) 要有效减少压力来源, 就必须在参与休闲运动方面 有良好的发展计划, 尤其是家庭休闲活动的实施更是刻不 容缓。因此, 有关部门应在各社区附近设置小型休闲运动 空间, 以增强大学生极其家庭参与休闲运动的交通便利性。

3) 休闲运动的良好发展, 与个人休闲运动技巧的指导 和整个环境方面的资源设施规划环环相扣, 缺一不可。本 研究发现大学生从事休闲运动会考虑自我本身运动技巧的 好坏。因此, 应建立合理的社会体育指导员的培训制度、 职业资格证书制度等, 提升社会体育事业的服务品质。学 校、班级组织大学生参与休闲运动, 则需配备专门的指导 人员, 让更多的大学生参与到休闲运动中来, 减少大学生 学习、生活中的压力。

\section{参考文献(References)}

[1] L.L. Yang. Study on social sources of stress and coping style of undergraduates. Theory review, 2010(4):48-49.

[2] X. Zhang. Investigation on the stressors of college students----A case study of Hubei University of Arts and Sciences. Journal of chifeng University (Chinese philosophy and social sciences Edition), 2012(10):244-246.

[3] L. Jang. Relationship between students' stress and cognitive errors. Psycho-social sciences, 2012(11):74-77.

[4] Iso-Ahola, S. E. Motivation for leisure. In E. J. Jackson \& T. L. Burton (Eds.), Understanding leisure and recreation: Mapping the past and charting the future State College, PA: Venture Publishing. pp. 247-279, 2005.

[5] Mull, R. F. \& Bayless, K. G. Recreational sports programming. Journal of Leisure Research, 21, pp.53-61, 2007.

[6] Mull, R. F., Bayless, K. G., Ross, C. M. \& Jamieson, L. M. Recreation sport management. IL: Human Kinetics, 2010. 